\title{
Anatomy of the lateral retinaculum
}

\section{Introduction}

The lateral retinaculum of the knee is not a distinct anatomic structure but is composed of various fascial structures on the lateral side of the patella. Anatomical descriptions of the lateral retinaculum have been published, but the attachments, name or even existence of its tissue bands and layers are controversial. The medial patellofemoral ligament on the other hand has been more recently re-examined and its detailed anatomy characterised (Amis et al., 2003, Nomura et al., 2005, Panagiotopoulos et al., 2006, Smirk and Morris, 2003, Tuxoe et al., 2002)

The first fascial layer is the fascia lata (deep fascia) that continues to envelop the knee from the thigh (Kaplan, 1957). The fascia lata covers the patellar region but does not adhere to the quadriceps apparatus. The iliotibial tract is integral to the deep fascia and is a lateral thickening of the fascia lata. The anterior expansion of the iliotibial band curves forward. It forms a group of arciform fibres and blends with the fascia lata covering the patella. Fulkerson (Fulkerson and Gossling, 1980) described the anatomy of the knee lateral retinaculum in two distinctly separate layers (Figure 1). The superficial oblique layer originates from the iliotibial band and interdigitates with the longitudinal fibres of the vastus lateralis. The deep layer consist of the deep transverse retinaculum with the epicondylopatellar ligament proximally and the patellotibial ligament distally. The patellotibial ligament proceeds obliquely to attach to the lateral meniscus and tibia. The epicondylopatellar ligament was said to be probably the same 
ligament described by Kaplan. Kaplan introduced the term epicondylopatellar ligament (superior retinaculum) and meniscopatellar ligament (inferior patellar retinaculum) (Kaplan, 1962). His diagram (Figure 2) depicts a low lying patella in which the epicondylopatellar ligament arising from the superior patella neither goes to the lateral femoral epicondyle nor the upper portion of the lateral femoral condyle but somewhere in between. It does not clearly define whether the epicondylopatellar ligament is derived from an expansion of the quadriceps apparatus or the capsule.

(Reider et al., 1981) described the lateral patellofemoral ligament as a palpable thickening of the joint capsule that connected the patella to the femoral epicondyles. Reider states that this was in agreement with Kaplan's description of the lateral epicondylopatellar ligament. However, both Fulkerson and Reider claimed agreement with Kaplan though in Fulkerson's case the band is extracapsular. Others have described differing versions of the lateral retinaculum. The longitudinal and horizonal components have been described in simple layers (Blauth and Tillmann, 1983) (Dye, 1993) (Figure 3), while others have described multiple layers (Terry et al., 1986) and complex interconnections (Seebacher et al., 1982) (Figure 3b) .

As regards to the anatomy of the lateral joint capsule in relation to the patellofemoral joint, this varies in the literature. Fulkerson and Gossling were of the opinion that the combined structure of the joint capsule and synovium was thin and provided little retinacular support (Fulkerson and Gossling, 1980). Some have described the complex arrangement of the joint capsule and recognised its role in tibiofemoral stability but no specific mention was made about the patellofemoral joint (Seebacher et al., 1982, Terry 
et al., 1986).Others have recognised their importance and described thickenings or reinforcements of the capsule as ligaments of various names (Blauth and Tillmann, 1983, Dye, 1993, Kaplan, 1962, Reider et al., 1981). Nonetheless, these ligaments vary in width and are absent in one third of knees (Blauth and Tillmann, 1983, Reider et al., 1981).

The description of the lateral retinaculum has been confusing because it is made up of condensations of tissue that intermix. In general, the studies concur that the deep fascia of the thigh goes over the patella and braces it, the iliotibial tract is a derivative of the deep fascia and by its expansions contributes to the retinaculum, the fibres of the quadriceps apparatus either directly or through aponeurotic expansions contribute to the retinaculum and that in the deeper layer there are transverse fibres that connect the underside of the iliotibial tract to the patella. The detail however is controversial. The sequence and exact arrangement of the layers and whether one layer is distinct from the next is contentious. The studies differ as regard to the attachments of the bands and raise the following questions. Is the epicondylopatellar ligament equivalent to the lateral patellofemoral ligament? What are its attachments? Is it a distinct enough a band and is it extracapsular or a thickening of the capsule. Similar issues also apply to the meniscopatellar, patellotibial and patellomeniscal "ligaments".

The aim of this study was to study the anatomy of the lateral restraints of the patella, to identify tissue bands that are morphologically important for further biomechanical investigation and to represent the anatomy of the anterolateral aspect of the patellofemoral joint in a manner useful for surgeons. 


\section{Materials and Method}

Thirty formalin fixed cadaveric knees and five fresh frozen knees were dissected. The skin and subcutaneous tissue was removed after identifying the deep fascia in the thigh from proximal to distal. Then, the deep fascial layer was incised longitudinally

proceeding distally in the midline over the patella. It was easily separated from the underlying muscle in the anterior part of the thigh and usually also from the deeper tissue overlying the patella. In the region of the patella and tibial tuberosity, abnormal thickening of the underlying bursae may cause adherence of the layers. Starting the incision over the patella or distal to the knee may cause one to lift more than one layer when the fascial incision is extended. In most cases, though, the tissue planes overlying the patella was more easily separated due to intervening bursa and tissue was reflected from the midline laterally in layers. Blunt dissection was used as much as possible to avoid inadvertently entering a deeper plane, however, in some areas cutting adherent tissue was necessary to display the anatomy.

Measurements were taken of patellar and soft tissue dimensions using a ruler. Tangential photographs were taken with a ruler incorporated in the photo for image analysis to verify measurements. The OsiriX medical imaging software (version 2.3 Digital imaging Unit University Hospital of Geneva) was used for this purpose. 


\section{Results}

The results of the anatomic dissection of the lateral retinaculum are presented below. An illustrative case showing a right knee will be used in the accompanying figures.

\section{Deep fascia}

The first layer deep to the skin and subcutaneous tissue was the deep fascia. The deep fascia passed over the patella. It was not attached to the patella and was easily separated from it, provided there were no abnormal thickenings of the pre-patellar bursa. Laterally, the deep fascia thickened to form the iliotibial tract in the lateral thigh. Deep to the deep fascia was the quadriceps muscle in the thigh. Distally as the muscle became the quadriceps tendon, there was an intervening layer, the quadriceps aponeurosis. The deep fascia is anchored to the quadriceps fascia proximal and lateral to the patella and

for a variable distance distally to the iliotibial tract. The deep fascia is incised in the midline and reflected laterally in (Figure $4 \& 5$ ).

\section{The quadriceps aponeurosis and iliotibial tract}

In Figures 4 and 5, the reflected deep fascia is held on its underside by a lateral extension of the quadriceps aponeurosis. The apex of this attachment is on average 28 $\mathrm{mm}$ (S.D. $11 \mathrm{~mm}$ ) proximal to the proximal edge of the patella and in line with the lateral edge of the patella. The attachment meets the deep fascia further lateral and distal. A surgeon approaching the lateral aspect of the knee in a subfascial approach from anteriorly will need to incise here to reflect the deep fascia further laterally. This attachment is just anterior to where the deep fascia condenses to become the iliotibial 
tract and occurs where the posterior part of the quadriceps aponeurosis and the anterior part of the iliotibial tract converge (Figure 6).

The bulk of the iliotibial tract fibres run in a longitudinal direction to the Gerdy's tubercle. The anterior fibres of the iliotibial tract curve anteriorly to meet the descending fibres of the quadriceps aponeurosis then descend with the quadriceps aponeurosis becoming indistinguishable from it. The most superficial of the iliotibial tract fibres, however, proceed obliquely across the iliotibial tract and then the quadriceps aponeurosis, being firmly adherent to both. They extend anteriorly up to the lower patella and the patellar tendon where they fuse with the quadriceps aponeurotic layer giving the quadriceps aponeurosis overlying the patellar tendon a more transverse appearance. In Figure 6 a probe is placed deep to these superficial fibres. In this specimen these fibres are thin and the underlying more dense fibres are seen. Kaplan named these fibres that cross obliquely on to and blending with the fascia of the patella as arciform fibres though he attributed these fibres to the more anterior fibres of the iliotibial tract (Kaplan, 1957). This has also been functionally called the iliopatellar band (Terry et al., 1986) and is likely to be analogous with the superficial oblique retinaculum (Fulkerson and Gossling, 1980).

The convergence of the descending fibres of the quadriceps aponeurosis along the lateral edge of the patella and the iliotibial tract is shown in Figure 6 and 7. 


\section{The vastus lateralis obliquus}

In Figure 8, a deep attachment between the underside of the iliotibial tract and the vastus lateralis obliquus tendon marks the proximal beginning of the deeper transverse fibres of the iliotibial tract. When the tendon can be followed all the way to the bony patella and this was the case in most knees, the tendon attaches close to the most lateral edge of the patella (where the patella is widest). In other knees, anatomical variation of the distal attachment made it impossible to tell if or where this attachment took place on the bony patella. The angle of pull (measured relative to the longitudinal axis of the patella) was on average 38 (S.D. $=11)$ degrees posterior, ranging from 20-60 degrees. The higher this angle, the higher the lateral force vector acting on the patella.

\section{The deeper transverse fibres of the iliotibial tract}

The deeper transverse fibres of the iliotibial tract are not a distinctly separate layer. Proximally, it is easily located by finding its attachment to the tendon of vastus lateralis obliquus by looking for a connection between the posterolateral edge of the vastus lateralis obliquus and the deep aspect of the iliotibial tract. From this point to uncover it further distally, the more longitudinal overlying fibres are removed by sharp dissection. To identify its distal extent, the longitudinal fibres of the iliotibial tract are split apart just proximal and anterior to Gerdy's tubercle. This split is continued proximally until resistance is met when transverse fibres are encountered. This is shown in Figure 9.

This "band" is not symmetrical and in this study it is measured from where it meets the oblique tendon and tangential to the mediolateral patellar axis. On average it was 37 
$\mathrm{mm}$ wide (proximal-distal) $(\mathrm{S} . \mathrm{D}=8)$ ranging from $18 \mathrm{~mm}$ to $52 \mathrm{~mm}$. The proximal edge where it meets the vastus lateralis obliquus can be located on average $20 \mathrm{~mm}$ (S.D. $=5$ (range $12-31 \mathrm{~mm}$ ) lateral to and $20 \mathrm{~mm}$ (S.D.=7 (range $7-32 \mathrm{~mm}$ ) proximal to the most lateral point of the patella. The deep transverse fibres of the iliotibial tract connect the iliotibial tract to the patella and the vastus lateralis. It does not attach to the lateral epicondyle of the femur.

\section{The lateral patellofemoral and the patellomeniscal ligament}

The joint capsule is thickened laterally to form the lateral patellofemoral ligament. This is variable from knee to knee in substance. The margins are difficult to define exactly as they represent a capsular thickening. The lateral patellofemoral ligament attaches the lateral patella to the lateral femoral epicondyle Figure 10. Similarly, a condensation of capsule connecting the inferolateral part of the patella to the meniscus was seen but for the same reason is difficult to quantify. These have been termed meniscopatellar (Blauth and Tillmann, 1983, Kaplan, 1957) or patellomeniscal (Dye, 1993). These supportive ligaments vary considerably and are not constantly found (Blauth and Tillmann, 1983, Kaplan, 1957, Reider et al., 1981).

\section{The patellotibial ligament}

The patellotibial band described by Fulkerson is in the same plane but distal to the deep transverse retinaculum and is said to attach from the distal part of the lateral edge of the patella to the meniscus and the underlying tibia (Fulkerson and Gossling, 1980). It is 
described as a distinct structure and synonymous with the meniscopatellar ligament. A patellotibial band as described could not be found in this study. The patellotibial ligament was described by Dye as often the same as the quadriceps aponeurosis layer (intermediate oblique layer) (Dye, 1993). This study was more in accordance with that description but in addition to that this layer also fuses anteriorly with the deeper joint capsule adjacent to and along the length of the patellar tendon.

\section{The lateral superior geniculate artery}

The lateral superior genicular artery arises from the popliteal artery posteriorly. It courses anteriorly towards the patella in roughly a horizontal direction between the capsule and the iliotibial tract. It remains deep to the iliotibial tract and is closely applied to the deep part of the deeper transverse fibres. It issues capsular branches distally and an oblique branch which travels on the superficial aspect of the distal part of the vastus lateralis (Figure 11). As the main artery nears the patella its relationship with the end of the oblique tendon of the vastus lateralis is variable. However, further anteriorly, as it crosses the edge of the patella, the artery always remained in the plane deep to the quadriceps aponeurosis and the continuation of the quadriceps tendon overlying the patella. The artery drifts proximally as it goes further anteriorly towards the proximal pole of the patella where it continues to the medial side and also meets a longitudinal branch which lies approximately in the midline of the patella. 
The quadriceps aponeurosis layer is easily separated from the quadriceps tendon in the prepatellar region but further laterally around the edge of the patella it begins to become adherent to deeper tissues as it interacts with the anterior fibres of the iliotibial tract. Therefore, a surgeon interested in protecting the artery from an outside-in lateral retinacular release can localise the artery beginning in the region overlying the patella, deep to the quadriceps aponeurosis layer. Then, following the vessel laterally past the lateral edge of the patella towards the area for tissue release. In fact as the quadriceps aponeurosis is thin overlying the patella, the vessel is visible through this layer, once the more superficial deep fascia is reflected off. In this manner, the artery can be located more easily than if the exploration starts laterally where the tissue is more dense and adherent.

Measurements were taken to relate the position of the artery at the lateral border of the patella. The distance from the proximal-distal equator of the patellar articular surface to the vessel at the lateral border of the patella was measured. On average, as the artery crosses the lateral edge of the patella it was $10 \mathrm{~mm}$ proximal to the proximal-distal equator of the articular surface of the patella. The standard deviation was $4.6 \mathrm{~mm}$ and the range was 0 to $16 \mathrm{~mm}$ proximal. A surgeon releasing the lateral retinaculum arthroscopically from within the joint will have to be cautious when he or she is proximal to this level. 


\section{Discussion}

The tissues, which restrain the patella, consist of the deep fascia in the most superficial layer and the joint capsule in the deepest layer. The deep fascia is not attached to the patella but is adherent to deeper tissues laterally thus acting as a brace. The most substantial structure is the iliotibial tract and its derivatives. The longitudinal fibres of the iliotibial tract merge with those of the quadriceps aponeurosis lateral and adjacent to the patella. This longitudinal retinaculum is adherent to the patella, the quadriceps tendon proximal to the patella and the joint capsule adjacent to the patellar tendon distal to the patella. These longitudinal fibres are reinforced by the horizontal retinaculum that consist of superficial fibres of the iliotibial tract (iliopatellar or arciform fibres) and the deeper transverse fibres of the iliotibial tract. The former cross obliquely over and adhere to the quadriceps aponeurosis overlying the patella and its tendon. The latter are dense and anchor the patella to the iliotibial tract and the oblique tendon of the vastus lateralis. This deeper transverse fibres band is not a distinctly separate layer.

Considering their location and fibre orientation, the deep transverse fibres may play an important role in lateral restraint of the patellofemoral joint. Although these transverse fibres are attached to the patella, they lack a direct connection to the femur and it can be argued that in this respect the lateral patellofemoral ligament which attaches to the lateral epicondyle of the femur may be more important. On the contrary, however, the former is more prominent and is consistently found. Moreover, this part of the iliotibial tract to which the deeper transverse band is attached, is relatively fixed in a longitudinal direction as a short segment. Proximally, it is anchored to the proximal aspect of the 
femoral condyle on average $8 \mathrm{~mm}$ (S.D. 7.1) proximal to the widest part of the patella and distally to Gerdy's tubercle of the tibia. In view of its longitudinal orientation, one would not expect iliotibial tract tension to affect patellofemoral tracking. However, when the bony fixation mentioned above and the transverse fibres are taken into account, it is likely that iliotibial tract tension plays a role in the functioning of the lateral retinaculum.

There are studies that lend support to this hypothesis. Two clinical studies report on the association between a tight iliotibial band and patellar maltracking (Puniello, 1993, Wu and Shih, 2004). A biomechanical study demonstrated that with loading of the iliotibial tract the patella translated laterally in most flexion angles shifting the patellofemoral contact area laterally (Kwak et al., 2000).

The study identifies three important new findings:

1) A connection of the deep fascia to the quadriceps tendon proximal and lateral to the patella via the lateral extention of the quadriceps aponeurosis (see Figure 4,5 $\& 12)$

2) The connection of the deeper transverse fibres to the tendon of the vastus lateralis obliquus (Figure 8).

3) The intimate relationship between the quadriceps aponeurosis and the iliotibial tract. The convergence of the longitudinal fibres of both structures and the fusion of the iliopatellar fibres (of the iliotibial tract) to the quadriceps aponeurosis overlying the patella and its tendon. 
The first finding will confer the deep fascia an anchorage and make it more effective as a brace for the patella. It is interesting that it is always present and it is a useful landmark for the surgeon to incise, localise the vastus lateralis obliquus and then to access the deeper transverse fibres of the iliotibial tract. This lateral extention of the quadriceps aponeurosis anchors the deep fascia close to where it thickens to form the iliotibial tract and thus (from proximal to distal) is the beginning of the interaction of the quadriceps aponeurosis and the iliotibial tract.

The connection of the deeper transverse fibres to the tendon of vastus lateralis obliquus is reminiscent of the interaction of the medial patellofemoral ligament with the vastus medialis obliquus muscle described in the literature (Amis et al., 2003, Panagiotopoulos et al., 2006). In addition to the direct attachment of the deeper transverse fibres to the patella, this connection affords an additional retinacular action by lateral pull of the tendon.

The lateral retinaculum is thus a complex structure that is difficult to delineate due to converging and interdigitating structures. The lateral retinaculum is principally an interaction of tissue interconnections of the various components of the iliotibial tract and the components of the quadriceps apparatus acting on the patella. They are composed of longitudinal fibres reinforced by horizontal fibres. There are also deeper capsular structures that link the patella to the femoral lateral epicondyle and to the lateral meniscus. 
To standardise anatomic descriptive terminology, the following scheme is suggested. The knee lateral retinacular complex is subdivided into superficial, intermediate and deep. These components consist of derivatives of fascia, the quadriceps apparatus and the capsule. The deeper transverse fibres of the iliotibial tract should be termed the transverse iliopatellar fibres. Accordingly, the iliopatellar (arciform) fibres are called the superficial iliopatellar fibres. The interactions of the various components are illustrated in Figure 12. A cross sectional diagram is shown in Figure 13. 
Figures 

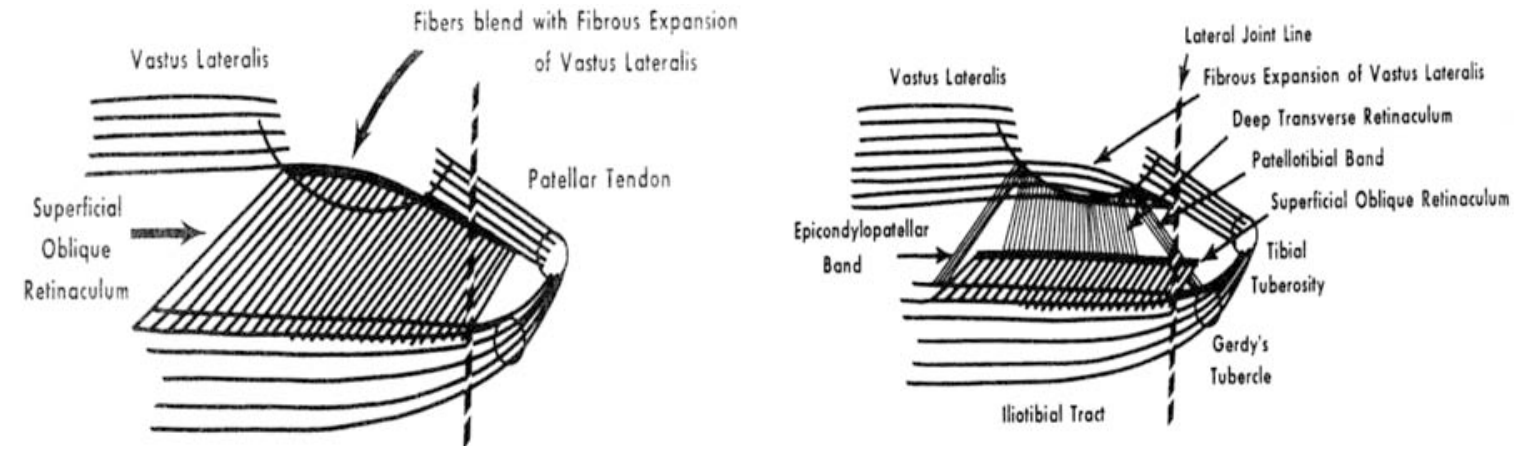

Figure 1. Diagram from Fulkerson and Gossling 1980. Superficial oblique retinaculum interdigitates with the vastus lateralis expansion on the patella (left). Deep to the superficial oblique retinaculum (right). 


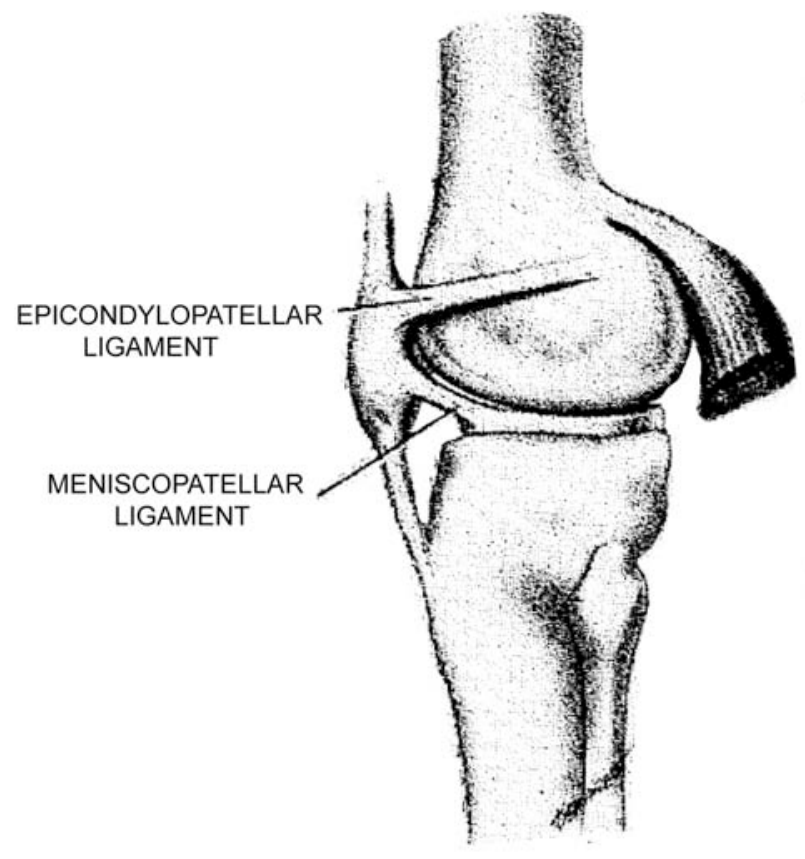

Figure 2. Diagram from Kaplan 1962. Lateral aspect of the left knee showing the superior patellar retinaculum (epicondylopatellar ligament) and the inferior patellar retinaculum (patellomeniscal reinforcement of the capsule) 


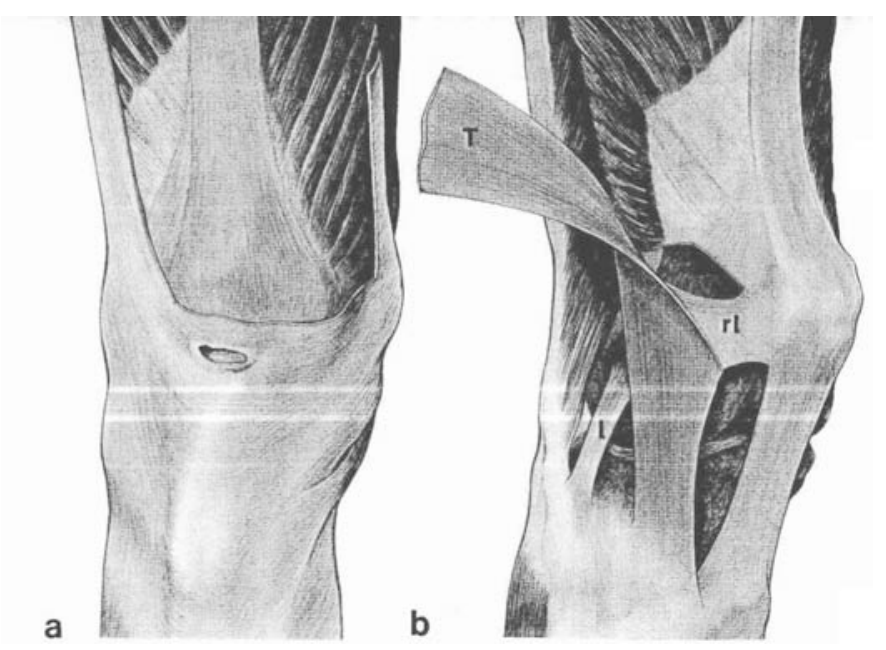

Figure 1. Drawing from Blauth and Tillmann (1983). Right knee joint, male 51 years old. a)The fascia lata was resected proximally: the nonresected distal part pass transversely across the patella. b) The iliotibial tract $(T)$ is cut and reflected laterally exposing the vastus lateralis muscle. The lateral horizontal retinaculum (rl) originates from the lateral patellar margin and inserts into the deep layers of the iliotibial tract. 


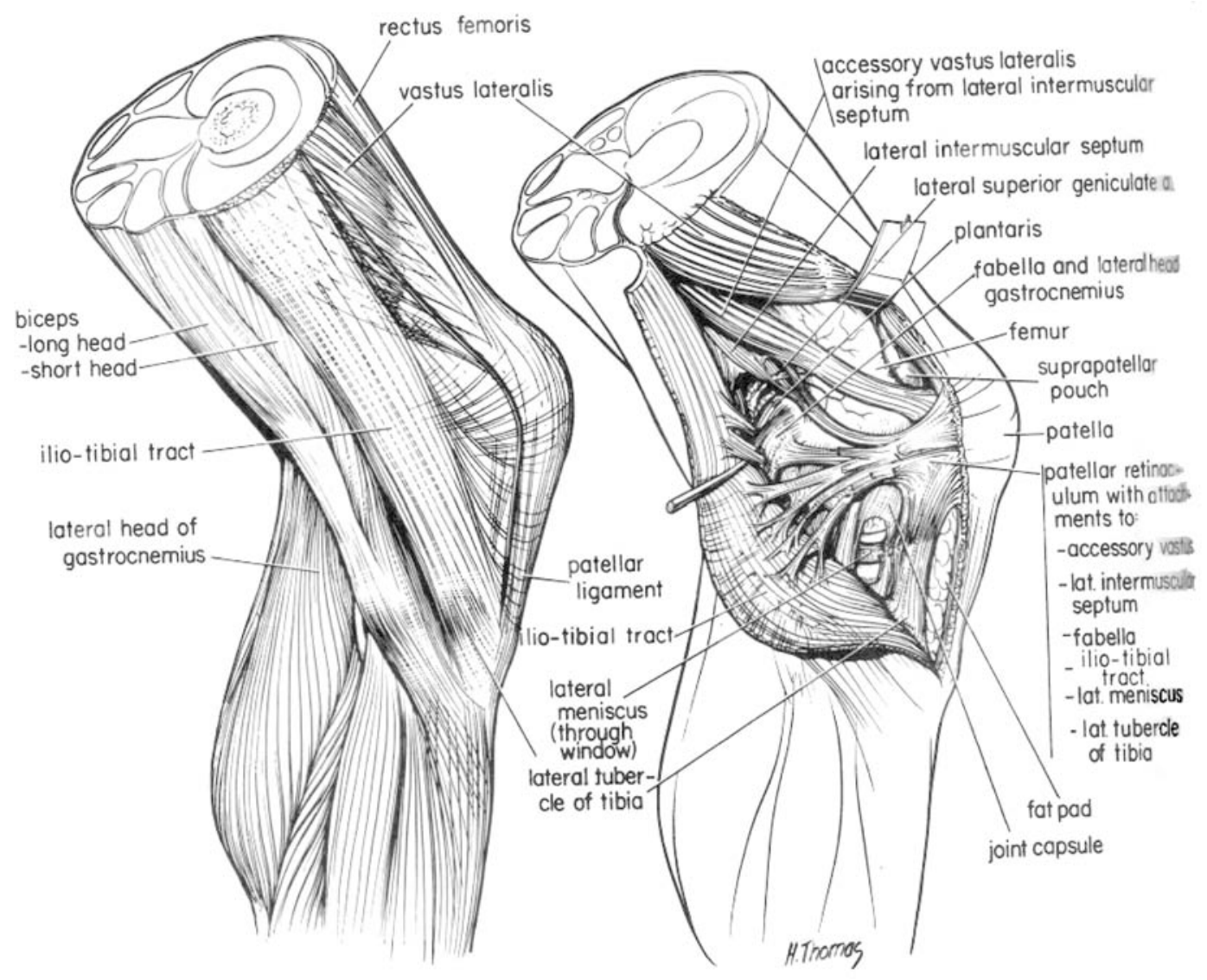

Figure 3b. Figure from Seebacher 1982. Drawing of the structures of the lateral side of the right knee depicting complex interconnections of the lateral retinaculum. 

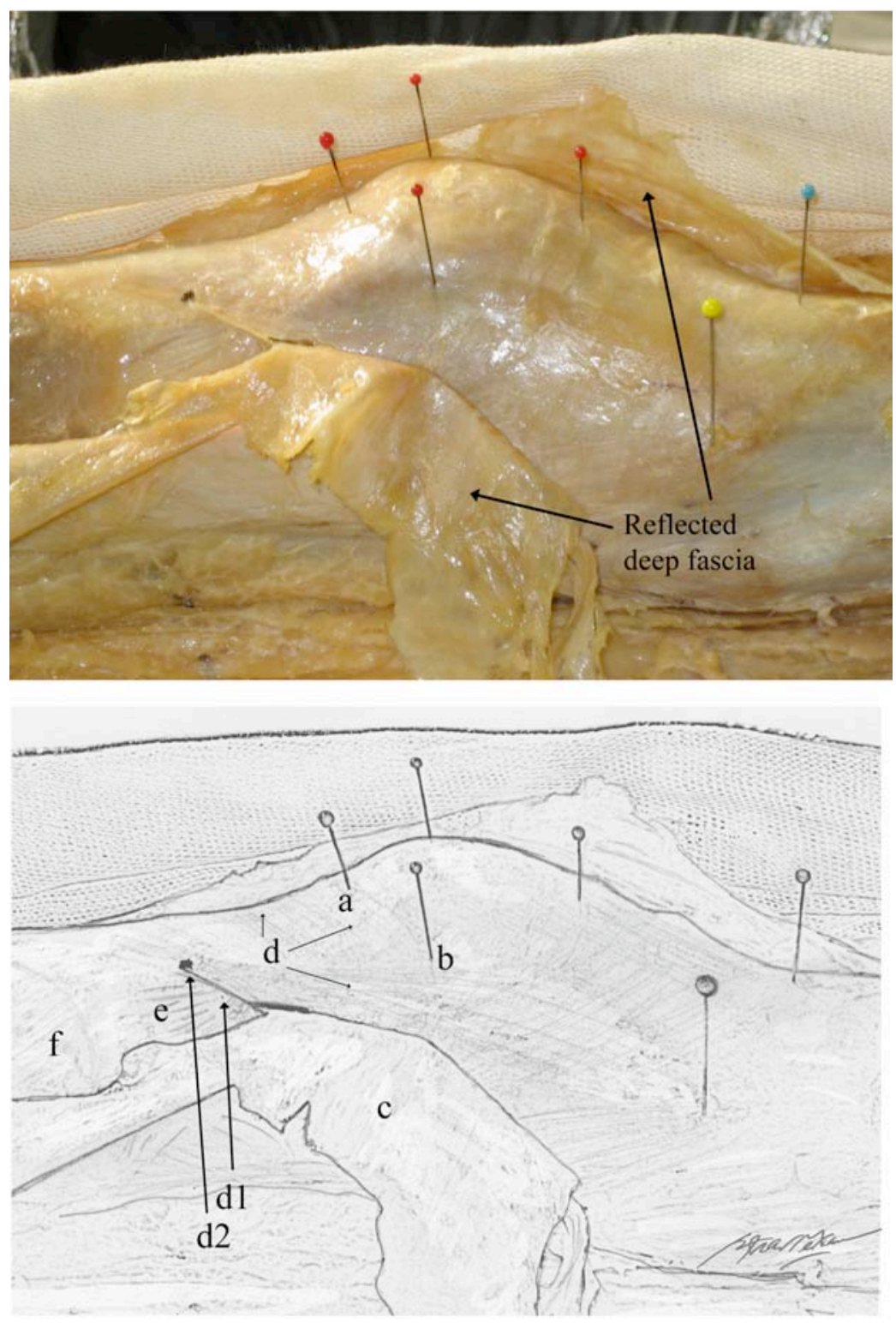

Figure 2. Deep fascia reflected. Lateral view. The proximal edge (a) and the lateral edge (b) of the patella. The deep fascia is reflected and the deep aspect is seen (c). The quadriceps aponeurosis (d) overlies the quadriceps tendon and continues over the region of the patella and also alongside the lateral edge of the patella (arrows). The lateral extension of the quadriceps aponeurosis (d1) anchors the deep fascia (c) and the apex of this attachment (d2) is adherent to the quadriceps tendon. A portion of the quadriceps tendon (e) and the vastus lateralis muscle (f) can be seen. 


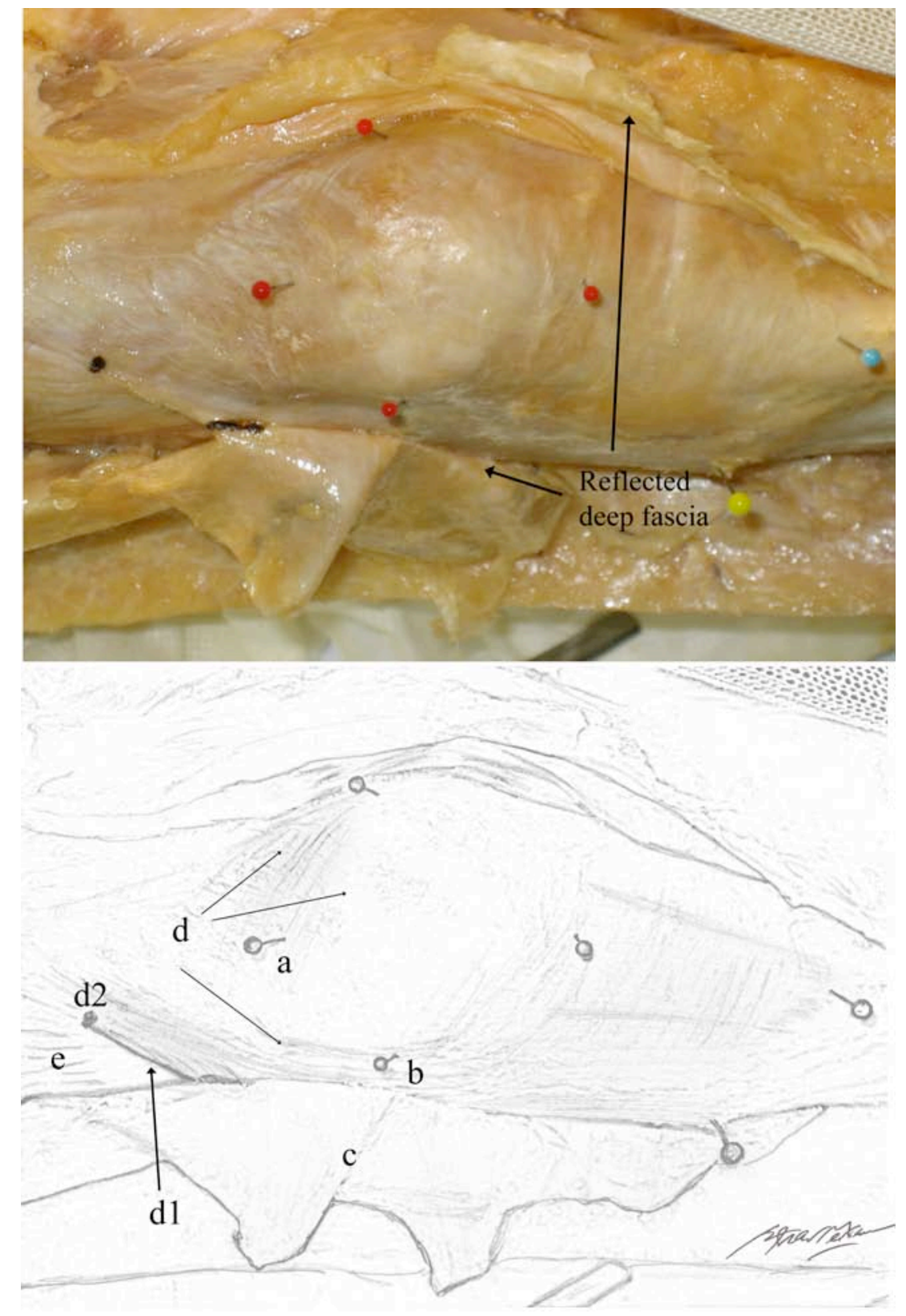

Figure 3. Deep fascia reflected. Anterior view. The proximal edge (a) and the lateral edge (b) of the patella. The deep fascia is reflected and the deep aspect is seen (c). The quadriceps aponeurosis (d) overlies the quadriceps tendon and continues over the region of the patella and also alongside the lateral edge of the patella (arrows). The lateral extension of the quadriceps aponeurosis (d1) anchors the deep fascia (c) and the apex of this attachment (d2) is adherent to the quadriceps tendon. A portion of the quadriceps tendon (e) can be seen. 


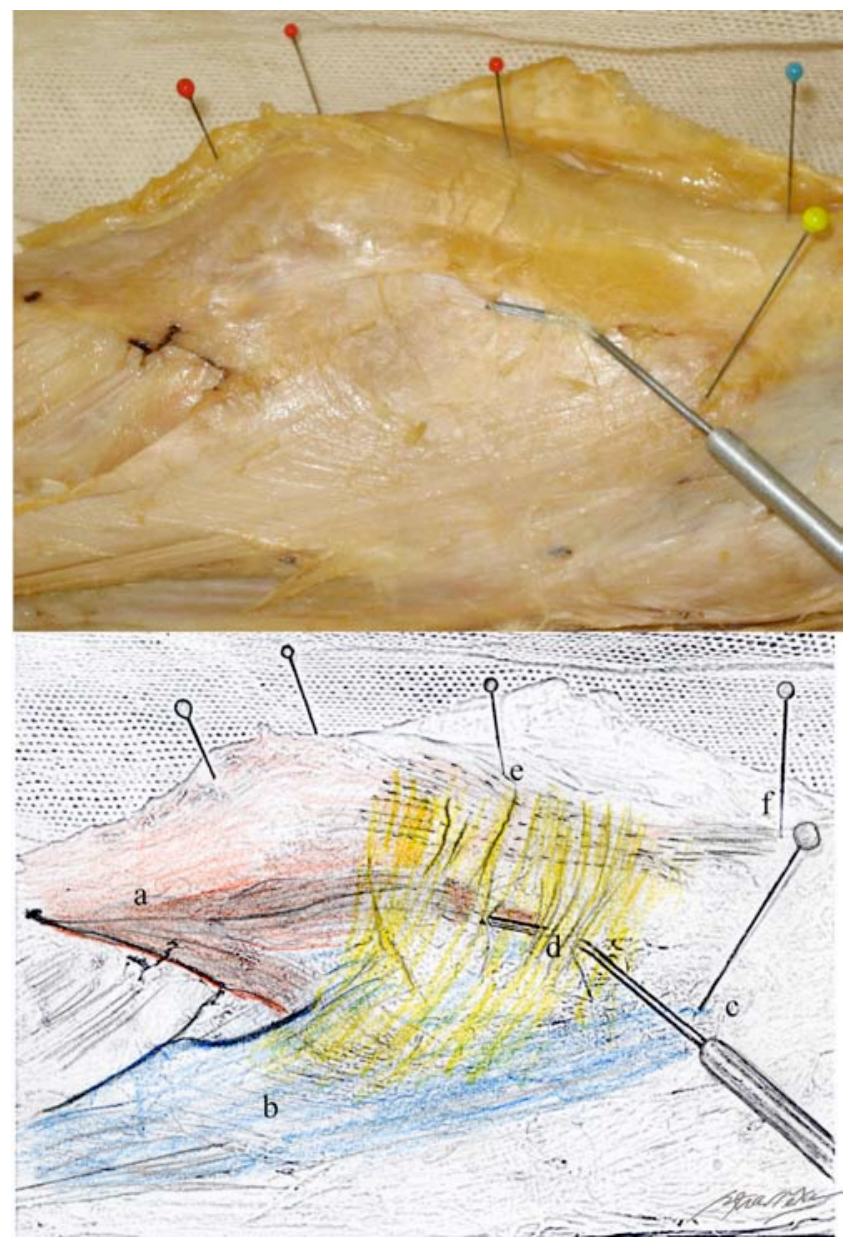

Figure 4. The iliotibial tract and quadriceps aponeurosis. Lateral view. The iliotibial tract (a) and quadriceps aponeurosis (b) converge. The main component of the iliotibial tract is longitudinal and attaches to the Gerdy's tubercle (c). The most superficial fibres of the iliotibial tract (d) sweep obliquely over the deeper iliotibial tract fibres then the quadriceps aponeurosis. In this specimen these superficial fibres of the iliotibial tract are relatively thin and are lifted up with a probe. These fibres pass superficially to the quadriceps aponeurosis and merges with it overlying the lower patella and the patellar tendon. Between the inferior edge of the patella (e) and the tibial tuberosity (f) the underlying patellar tendon can be seen. 

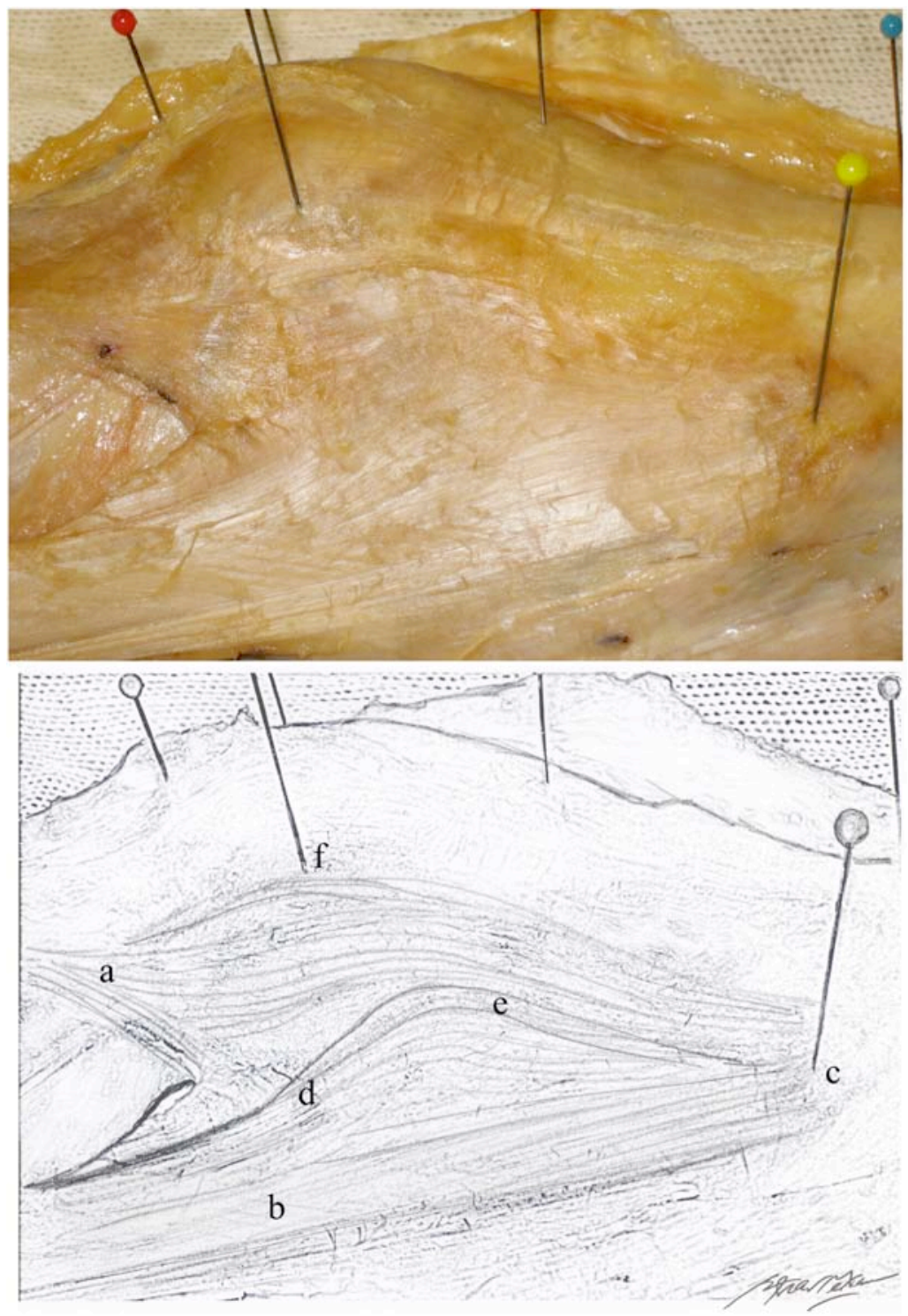

Figure 5. The iliotibial tract and quadriceps aponeurosis. Lateral view. The superficial fibres of the iliotibial tract in the previous figure has been removed. The descending fibres of the quadriceps aponeurosis (a) and the iliotibial tract (b) converge. The main component of the iliotibial tract attaches to Gerdy's tubercle (c). The more anterior fibres curve anteriorly (d) then descend slightly posteriorly (e) alongside the quadriceps aponeurosis becoming indistinguishable from it. Lateral edge of patella (f). 


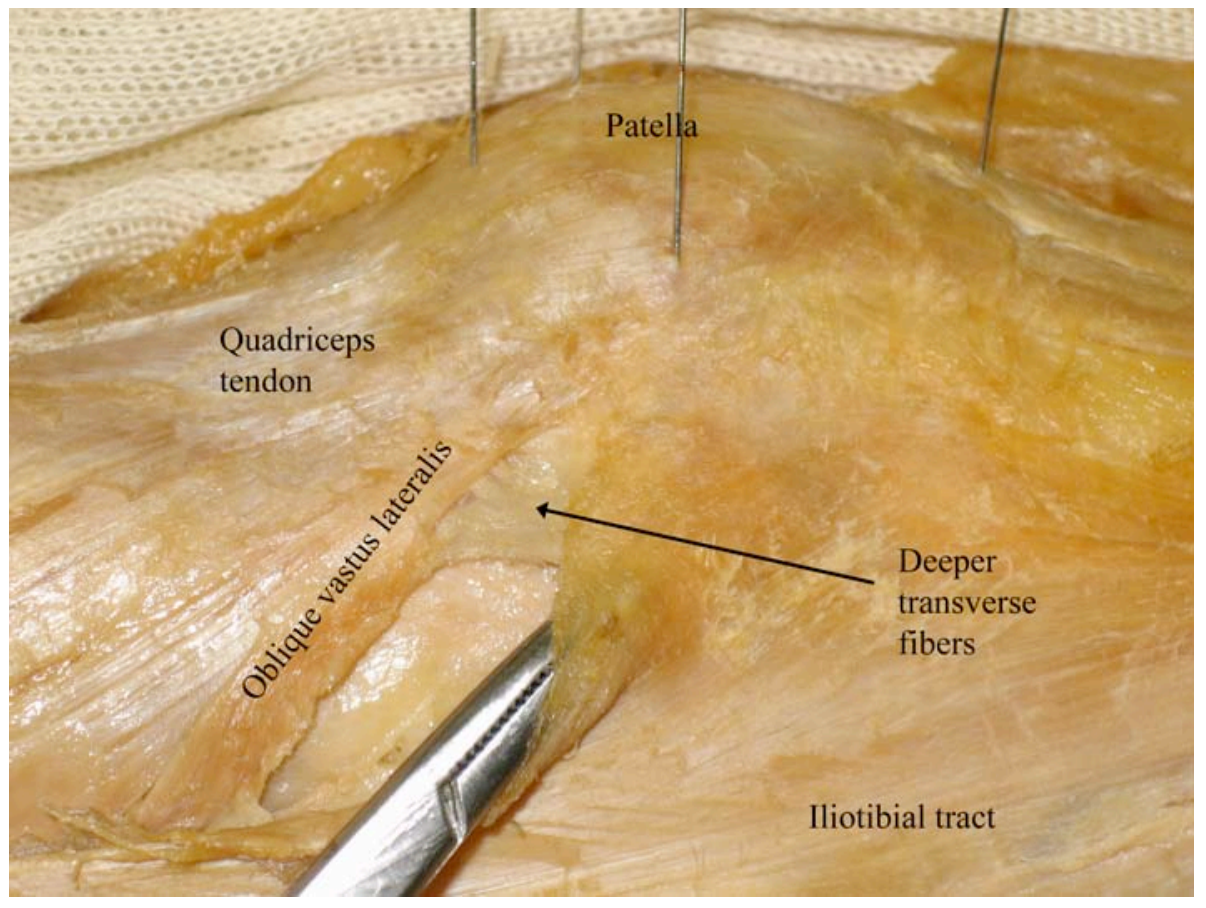

Figure 6. The oblique vastus lateralis tendon and the attachment of the deeper transverse fibers to it. 

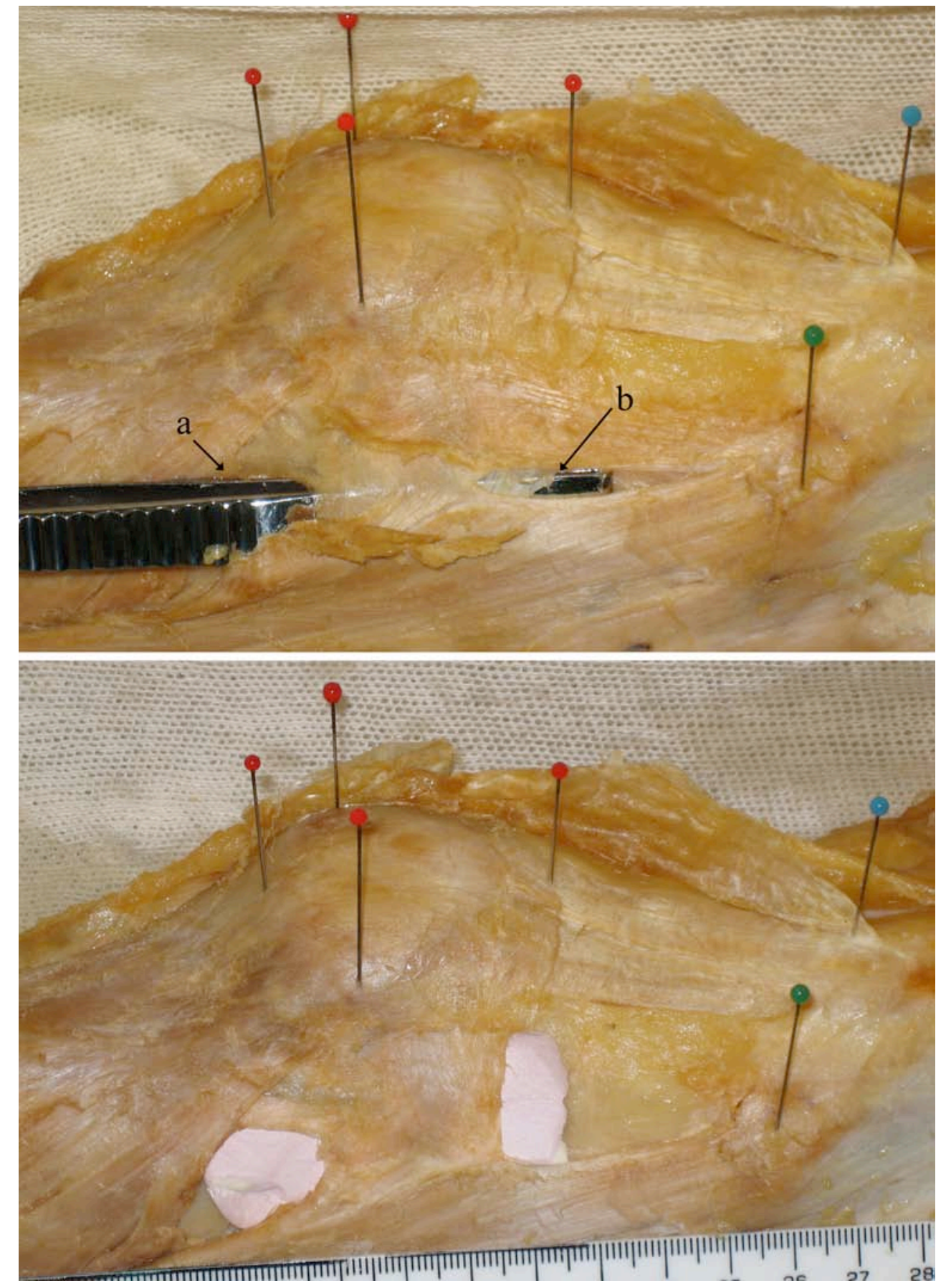

Figure 7. The deep transverse fibres of the iliotibial tract. In the top picture the proximal connection to the oblique tendon is identified (as described in the text) and the distal limit is found by splitting the longitudinal fibres from distal to proximal, along the line of the forceps. The bottom picture is after removal of the superficial fibres. 

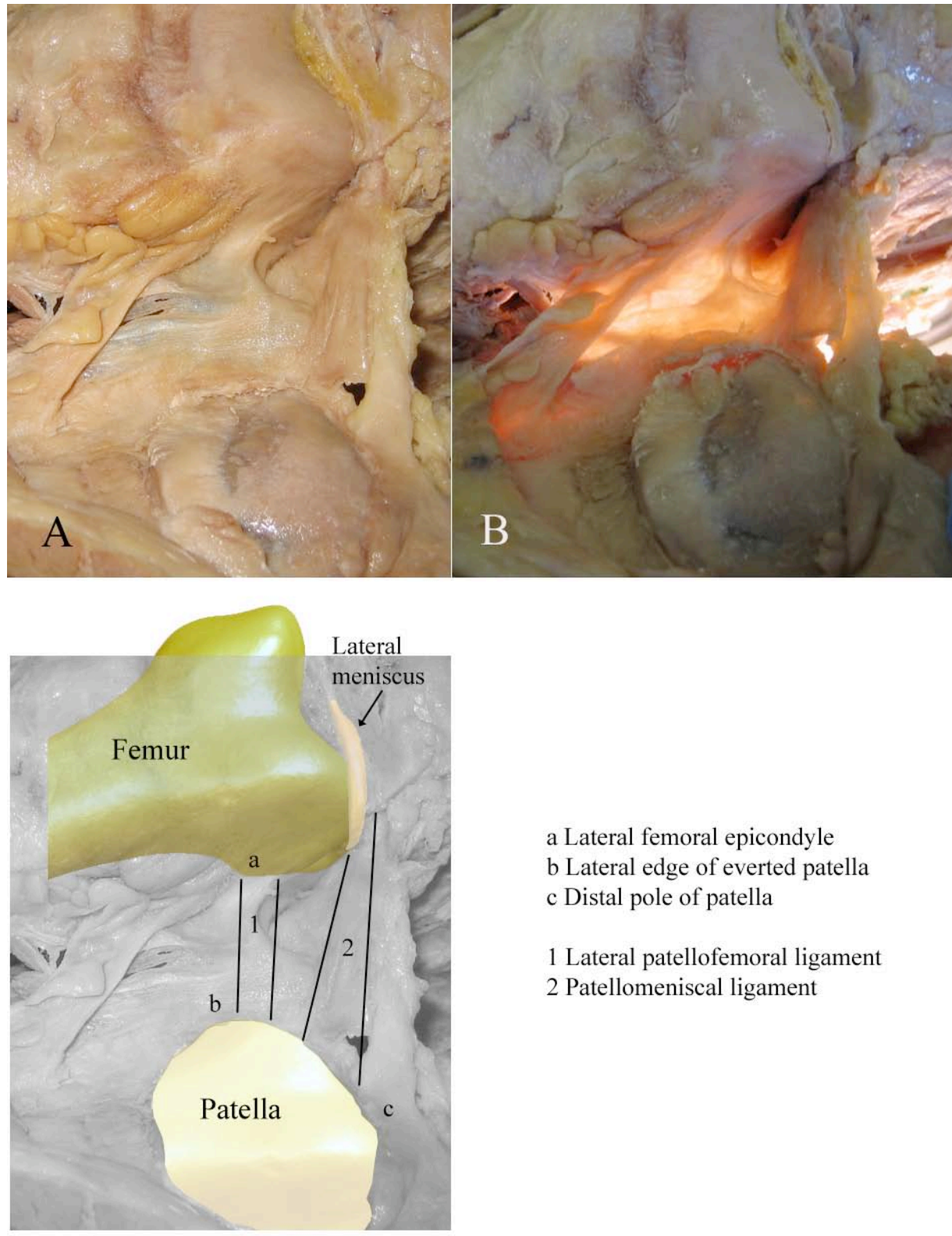

a Lateral femoral epicondyle

b Lateral edge of everted patella

c Distal pole of patella

1 Lateral patellofemoral ligament

2 Patellomeniscal ligament

Figure 8. The lateral patellofemoral and patellomeniscal ligament. The right knee joint is opened medially and the patella is everted to view the lateral knee capsule from within (A). (B) is the same view with a light source placed to transiluminate the capsule. Thickenings of the capsule form these ligaments. 


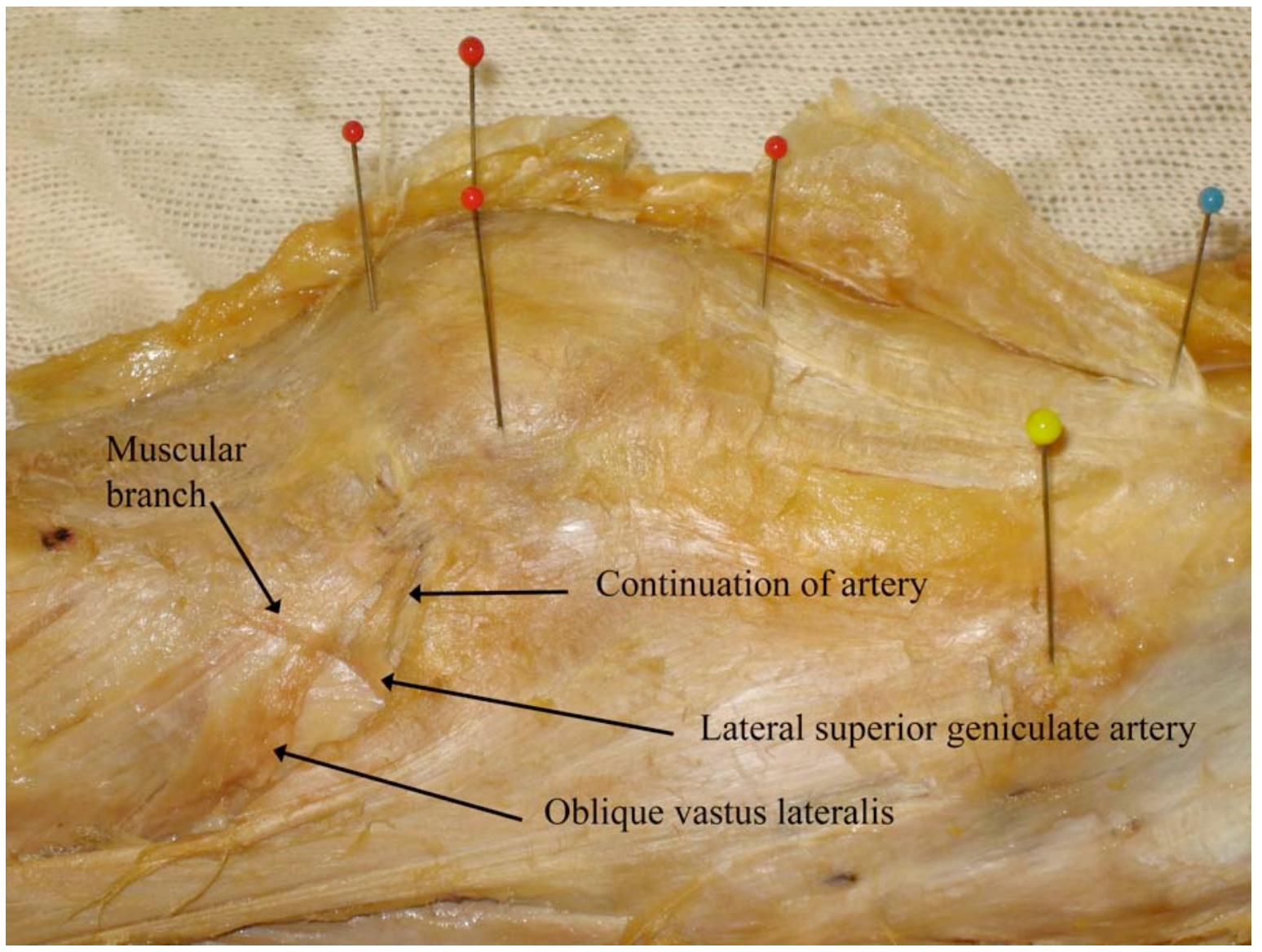

Figure 01. The lateral superior genicular artery. The quadriceps aponeurosis overlying the patella, the quadriceps tendon and the patellar tendon has been removed. The lateral superior genicular artery from the underside of the iliotibial tract courses anteriorly towards the patella. It issues a oblique branch which passes over the vastus lateralis muscle. The oblique vastus lateralis is shown arising from the lateral intermuscular septum. 


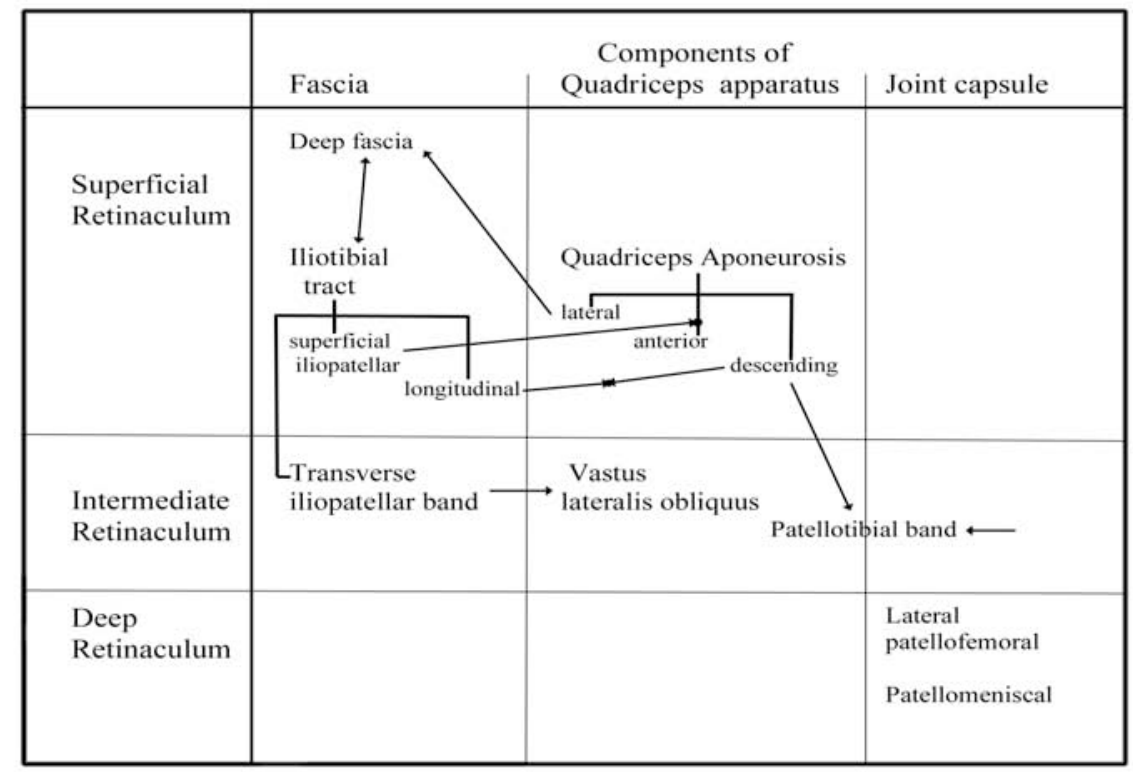

Figure 9. The knee lateral retinacular complex. The retinacular complex consists of the superficial, intermediate and deep parts. These consist of components of the fascia, quadriceps apparatus and joint capsule. Interconnections and attachments are shown as arrows. The deeper transverse fibres of the iliotibial tract have been renamed the transverse iliopatellar band. 


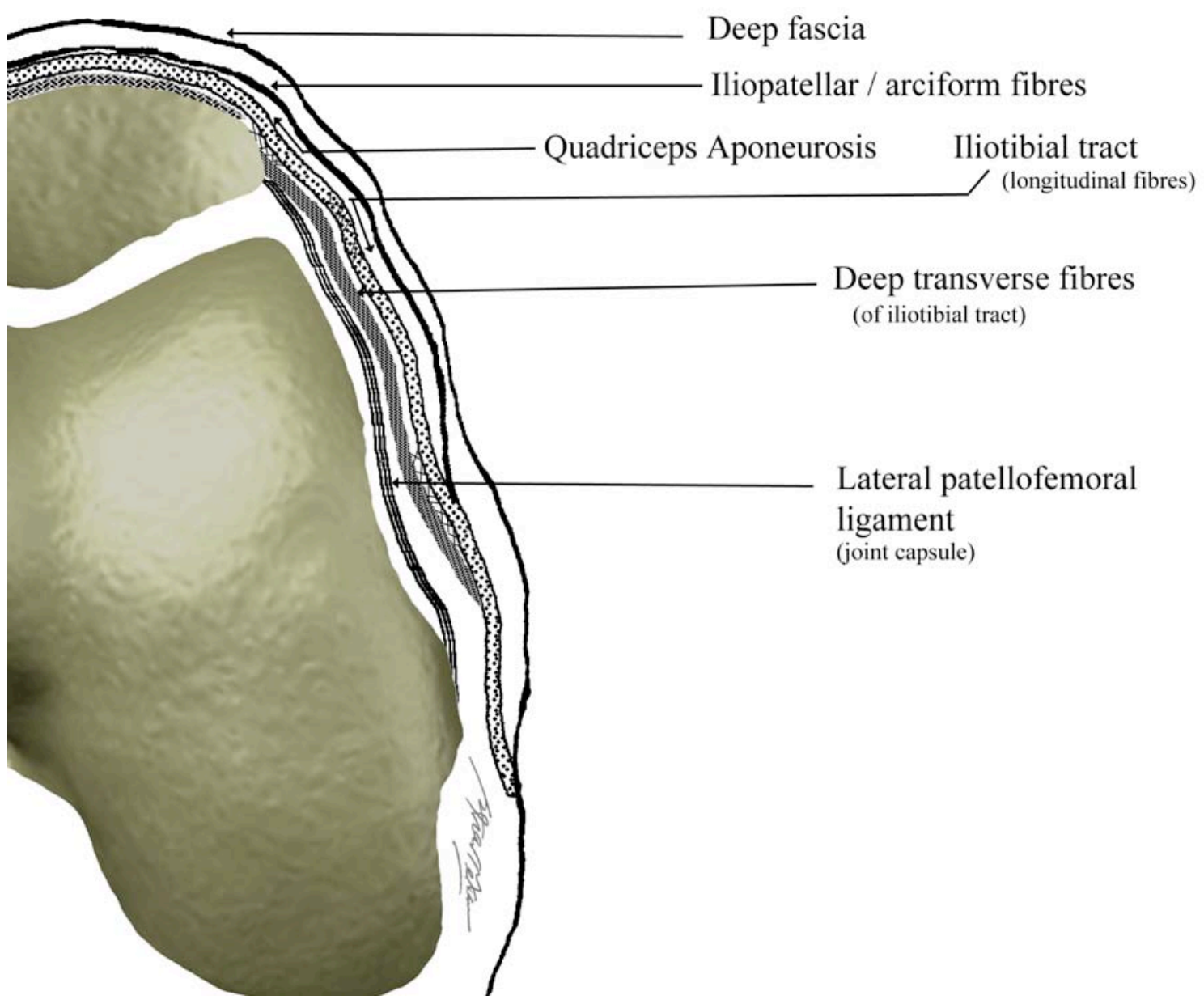

Figure 13. The lateral retinaculum of the knee. A cross sectional diagram illustrating the components of the lateral retinaculum of the knee. The cross section is taken at the level of the lateral femoral epicondyle. 


\section{References}

AMIS, A. A., FIRER, P., MOUNTNEY, J., SENAVONGSE, W. \& THOMAS, N. P. (2003) Anatomy and biomechanics of the medial patellofemoral ligament. Knee, $10,215-20$.

BLAUTH, M. \& TILLMANN, B. (1983) Stressing on the human femoro-patellar joint. I. Components of a vertical and horizontal tensile bracing system. Anat Embryol (Berl), 168, 117-23.

DYE, S. F. (1993) Chapter 1: Patellofemoral anatomy. In: Fox IM, Del Pizzo W, eds. The Patellofemoral Joint, 3-6.

FULKERSON, J. P. \& GOSSLING, H. R. (1980) Anatomy of the knee joint lateral retinaculum. Clin Orthop Relat Res, 183-8.

KAPLAN, E. B. (1957) Surgical approach to the lateral (peroneal) side of the knee joint. Surg Gynecol Obstet, 104, 346-56.

KAPLAN, E. B. (1962) Some aspects of functional anatomy of the human knee joint. Clin Orthop, 23, 18-29.

KWAK, S. D., AHMAD, C. S., GARDNER, T. R., GRELSAMER, R. P., HENRY, J. H., BLANKEVOORT, L., ATESHIAN, G. A. \& MOW, V. C. (2000)

Hamstrings and iliotibial band forces affect knee kinematics and contact pattern. Journal of Orthopaedic Research, 18, 101-108.

NOMURA, E., INOUE, M. \& OSADA, N. (2005) Anatomical analysis of the medial patellofemoral ligament of the knee, especially the femoral attachment. Knee Surg Sports Traumatol Arthrosc, 13, 510-5.

PANAGIOTOPOULOS, E., STRZELCZYK, P., HERRMANN, M. \& SCUDERI, G. (2006) Cadaveric study on static medial patellar stabilizers: the dynamizing role of the vastus medialis obliquus on medial patellofemoral ligament. Knee Surg Sports Traumatol Arthrosc, 14, 7-12.

PUNIELLO, M. S. (1993) Iliotibial Band Tightness and Medial Patellar Glide in Patients with Patellofemoral Dysfunction. Journal of Orthopaedic \& Sports Physical Therapy, 17, 144-148.

REIDER, B., MARSHALL, J. L., KOSLIN, B., RING, B. \& GIRGIS, F. G. (1981) The anterior aspect of the knee joint. J Bone Joint Surg Am, 63, 351-6.

SEEBACHER, J. R., INGLIS, A. E., MARSHALL, J. L. \& WARREN, R. F. (1982) The structure of the posterolateral aspect of the knee. J Bone Joint Surg Am, 64, 536-41.

SMIRK, C. \& MORRIS, H. (2003) The anatomy and reconstruction of the medial patellofemoral ligament. Knee, 10, 221-7.

TERRY, G. C., HUGHSTON, J. C. \& NORWOOD, L. A. (1986) The Anatomy Of The Iliopatellar Band And Iliotibial Tract. American Journal Of Sports Medicine, 14, $39-45$.

TUXOE, J. I., TEIR, M., WINGE, S. \& NIELSEN, P. L. (2002) The medial patellofemoral ligament: a dissection study. Knee Surg Sports Traumatol Arthrosc, 10, 138-40.

WU, C. C. \& SHIH, C. H. (2004) The influence of iliotibial tract on patellar tracking. Orthopedics, 27, 199-203. 